\title{
Construcción y validación de una Escala de actitud hacia la matemática para estudiantes de psicología*
}

\section{Construction and Validation of an Attitude-Toward-Mathematics Scale for Psychology Students}

\author{
Facundo Juan Pablo Abat* \\ Consejo Nacional de Investigaciones Científicas y \\ Técnicas (CONICET), Argentina \\ ORCID: http://orcid.org/0000-0001-7023-5380 \\ Sofía Esmeralda Auné \\ Consejo Nacional de Investigaciones Científicas y \\ Técnicas (CONICET), Argentina \\ ORCID: http://orcid.org/0000-0002-0620-0199 \\ Horacio Félix Attorresi \\ Universidad de Buenos Aires, Argentina \\ ORCID: http://orcid.org/0000-0002-3027-1069
}

\footnotetext{
* Autor de correspondencia. Correo electrónico: fabal@psi.uba.ar
}

Para citar este artículo: Abal, F. J. P., Auné, S. E., \& Attorresi, H. F. (2018). Construcción y validación de una escala de actitud hacia la matemática para estudiantes de psicología. Universitas Psychologica, 17(4), 1-15. https://doi.org/10.11144/Javeriana.ups y17-4.cvea

\section{RESUMEN}

El objetivo de este trabajo es presentar el proceso de construcción de una escala de actitud hacia la matemática específica para estudiantes de Psicología. Se tomaron como referencia las dimensiones del constructo más mencionadas en la literatura y se generaron ítems para operacionalizarlas. Se efectuó una depuración mediante juicio experto y estudio piloto. Los ítems conservados se administraron una muestra de 2814 estudiantes. Mediante un análisis factorial exploratorio, se identificaron tres dimensiones relevantes (Afectivo-motivacional, Confianza y Utilidad) que fueron replicadas en un estudio confirmatorio. Este resultado aportó evidencias de validez de constructo. Se obtuvieron indicadores óptimos de consistencia interna y estabilidad temporal. Se compara la estructura factorial alcanzada con las reportadas en otras investigaciones que miden el mismo constructo.

\section{Palabras clave}

actitud hacia la matemática; afecto hacia la matemática; confianza para la matemática; utilidad de la matemática; estudiantes de Psicología.

\begin{abstract}
The aim of this work was to present the construction process of an attitude-toward-mathematics scale for students of Psychology. The most widely reported dimensions of the construct in literature were taken as reference and items were generated to make these dimensions operational. Expert judgment and a pilot test were used to reduce the number of items. The items accepted were administered to a sample of 2814 students. Three relevant dimensions were identified using exploratory factor analysis (affection-motivation, confidence and usefulness), which were replicated in a confirmatory study. This result provided evidence of construct validity. Optimal indicators of internal consistency and temporal stability
\end{abstract}


were obtained. The factor structure obtained is comparable to those reported in other studies measuring the same construct.

Keywords

attitude toward mathematics; affection toward mathematics; confidence in mathematics; usefulness of mathematics; psychology students.

El estudio de la actitud hacia la matemática ha despertado interés por parte de los investigadores del campo educativo en todos sus niveles, porque constituye una herramienta analítica útil para evaluar la eficacia del proceso de aprendizaje y las potenciales innovaciones introducidas al mismo. Dentro del ámbito universitario, resulta fundamental no descuidar la posible interacción entre la carrera y la actitud de los estudiantes hacia la Matemática (Gómez-Chacón, 2009; Pérez-Tyteca, 2012). Los alumnos que cursan carreras de ciencias físico-químico-matemáticas o tecnológicas aceptan los contenidos como una parte necesaria de su formación, a fin de cimentar futuros conocimientos (Álvarez \& Ruiz, 2010). Sin embargo, una parte considerable de los estudiantes de áreas sociales o del comportamiento tienden a manifestar abiertamente su rechazo hacia materias de corte cuantitativo (Blanco, 2004; Narro, 1997; Rodríguez Feijóo, 2011; Ruiz de Miguel, 2015). Los ingresantes a estas carreras suelen inscribirse suponiendo que se trata de carreras nomatemáticas y se asombran al descubrir que deben cursar materias que requieren habilidades para el cálculo (Coolican, 1994; Cortada, Rodríguez Feijóo, \& Kohan Cortada, 2008). Desde el punto de vista de los docentes, se pone de manifiesto la necesidad de adecuar u omitir las demostraciones matemáticas para evitar el rechazo de la materia (Carpintero, Garriga Trillo, Ponsoda \& Yela, 1989). Así también, se debe estar atento a otras condiciones adversas propias de un nivel universitario como los cursos masivos y la escasez de tiempo. Frente a este panorama, el desafío de la docencia está más allá de la transmisión de los contenidos y obliga a intervenir sobre la componente afectiva del aprendizaje en pos de alcanzar la cabal comprensión de los conceptos.
Se han desarrollado instrumentos para la evaluación de la actitud hacia la matemática o estadística en población universitaria general (Rodríguez Feijóo, 2011; Rodríguez Jiménez \& Mora Mojica, 2016). Pero las muestras de estandarización no resultan lo suficientemente exhaustivas ni existen referencias que avalen la invarianza de la medida en función de la carrera. En este sentido, podría resultar riesgoso aceptar que los indicadores utilizados para la medición en una carrera pueden ser igualmente descriptivos para otra, perdiendo así capacidad discriminativa. Con mayor frecuencia, se observa que los investigadores acotan el estudio del constructo a poblaciones de carreras donde existe un fuerte impacto de la matemática (Álvarez \& Ruiz, 2010; Bazán \& Sotero, 1998; Lim \& Chapman, 2013).

La relación particular que tienen los estudiantes de carreras como Psicología, Sociología y Ciencias de la Educación con la matemática obliga a pensar en la necesidad de generar instrumentos que permitan detectar con mayor detalle las diferencias individuales propias de esta población. El uso de la metodología cuantitativa en la construcción del conocimiento científico de estas disciplinas ha mostrado un avance considerable en las últimas décadas. En consecuencia, resulta necesario profundizar en las variables intervinientes en los procesos formativos de estas áreas con el fin de aportar elementos para la elaboración de estrategias didácticas más específicas (Blanco, 2004; Rodríguez Feijóo, 2011).

El objetivo de esta investigación es elaborar una escala que permita medir la actitud hacia la matemática para universitarios de carreras que tradicionalmente experimentan rechazo o indiferencia por el conocimiento matemático. En los siguientes apartados se reseñan los referentes conceptuales que describen el marco teórico usado para la construcción del instrumento. 


\section{La actitud hacia la matemática en el dominio afectivo de la enseñanza}

Las características inherentes de la matemática suscitan que las personas vinculen frecuentemente a esta disciplina con aspectos cognitivos como la capacidad de abstracción y el razonamiento lógico. No obstante, en el aprendizaje de sus nociones hay involucrado un monto de afectividad considerable ocasionado por las habilidades que requieren, los métodos de enseñanza usados y los docentes que las enseñan (Gómez Chacón, 2000; McLeod, 1992). En las últimas décadas, ha crecido la investigación para precisar esta dimensión no cognitiva, tanto desde una vertiente teórica como su transferencia a las aplicaciones en el aula (Gairín, 1990; Hernández, 2011; McLeod, 1992). En estos estudios, se denomina dimensión afectiva a un extenso rango de sentimientos y estados de ánimo que intervienen en los procesos de enseñanza, pero son considerados como una entidad diferente a la pura cognición (Gómez-Chacón, 2000).

Los estudios iniciales sobre la afectividad en el aprendizaje de la matemática recaían sobre las actitudes como concepto central. Concretamente, la actitud hacia la matemática se introdujo a mediados del siglo XX como una nueva variable útil para explicar el fracaso académico de los estudiantes, aun a expensas de su normal desempeño cognitivo (Hernández, 2011). Estos desarrollos fueron predominantemente psicométricos (Aiken, 1974; Fennema \& Sherman, 1976), y su objetivo principal se centró en determinar si las diferencias individuales en la actitud mostraban incidencia en el rendimiento de los alumnos.

Durante ese período, se encuentra una distinción conceptual sugerida por Aiken y Aiken (1969) entre las actitudes hacia la ciencia y las actitudes científicas que fue retomada por numerosos autores para categorizar los objetos actitudinales asociados a la matemática (Gómez-Chacón, 2000; Hidalgo, Maroto, \& Palacios, 2005; Martínez, 2008). La actitud hacia la matemática remite a un conjunto de manifestaciones afectivas que despiertan una aproximación hacia esta disciplina. En esta categoría, la matemática queda ubicada como objeto actitudinal y el interés está puesto en identificar la valoración, satisfacción e interés que el individuo tiene tanto por la disciplina científica como por su aprendizaje. Las actitudes matemáticas, en cambio, presentan un marcado componente cognitivo, en tanto que describen un conjunto de capacidades mentales necesario para la actividad matemática (i. e., flexibilidad de pensamiento, objetividad, pensamiento crítico, entre otras). Este desdoblamiento de los componentes involucrados en el proceso de enseñanza aprendizaje abrió la posibilidad de contraponer a la dimensión cognitiva reconocida tradicionalmente una dimensión no cognitva.

Durante la década de 1990 se observó el surgimiento y consolidación de un paradigma alternativo para la comprensión de la dimensión afectiva basado en la fusión de los aportes de la psicología cognitiva y del socioconstructivismo (Goldin, 1988; Gómez-Chacón, 2010; McLeod, 1992). Desde esta perspectiva, se intentó focalizar en el análisis de las conductas del alumno y en las reacciones emocionales que despierta la matemática. McLeod (1992) identificó tres descriptores básicos de la dimensión afectiva (creencias, actitudes y emociones) y caracterizó sus interrelaciones a partir de la intensidad y estabilidad temporal. En este modelo, las actitudes se presentan como un puente entre las creencias, que presentan poca intensidad pero fuerte estabilidad en el tiempo, $y$ las emociones, que poseen gran intensidad pero no son estables (Di Martino \& Zan, 2011). Así también, establece un solapamiento entre los constructos, dado que las actitudes incluyen una componente cognitiva (producto de la influencia de las creencias) y una afectiva (originada por las emociones).

Más recientemente, la evolución de la conceptualización del dominio afectivo se volcó a tomar mayor consideración del contexto de aprendizaje. El estudiante participa activamente en el proceso de construcción del conocimiento matemático y sus acciones se ven influenciadas por las creencias de sus docentes, compañeros, padres y por la de otros actores del sistema educativo y social. Esto conlleva no reducir 
la actitud a un aspecto propio del estudiante, sino que además se incorporan en el análisis las interacciones que se producen entre los actores involucrados (Gómez-Chacón, 2000; Grootenboer \& Marshman, 2016; Harkness, D'ambrosio, \& Morrone, 2007). El factor social constituye un importante peso en la formación y mantenimiento de la actitud hacia la matemática, debido a que frecuentemente las actitudes y las creencias son compartidas con las personas que forman parte de este contexto (Martínez, 2008).

\section{Definiciones de la actitud hacia la matemática}

A pesar de los avances generados a lo largo de su historia, la actitud hacia la matemática presenta un problema insoslayable: no existe una teoría que haya alcanzado a unificar estos desarrollos teóricos (García-González \& Juárez-López, 2011). Un gran número de investigaciones desarrolladas en torno a este constructo suelen estar plagadas de equívocos $\mathrm{y}$ dificultades que redundan en resultados inconsistentes o incomparables. Esta ausencia de una delimitación conceptual clara y consensuada dificulta conocer el aporte real de las actitudes a la dimensión afectiva.

Di Martino y Zan (2015) sistematizaron dos líneas principales sobre las que se pueden clasificar las definiciones teóricas sobre la actitud hacia la matemática, registradas en la literatura. Una de ellas caracteriza a la actitud como un fenómeno que puede ser representado con un modelo unidimensional, cuyos extremos oscilan entre sentimientos de agrado hasta desagrado (Brito, 1998; Haladyna, Shaughnessy, \& Shaughnessy, 1983; McLeod, 1992). Las actitudes son una reacción eminentemente afectiva/emocional frente a un objeto matemático. En la misma postura, Gómez Chacón (2000) se refirió a la actitud hacia la matemática como a una predisposición evaluativa (positiva o negativa) que determina una intencionalidad en el alumno para aproximarse a ella o rechazarla. La segunda corriente desestima una definición simple y defiende una estructura compleja multidimensional que contempla las componentes cognitivo, afectivo y comportamental (Auzmendi, 1992; Bazán \& Sotero, 1998; Hart, 1989; Neale, 1969).

Ninguna de las dos posturas está exenta de cuestionamientos: la visión unidimensional por su excesiva simplificación del fenómeno y la visión tricomponencial por la dificultad para obtener sustento empírico (Sánchez Mendías, 2013). La importancia relativa otorgada a cada una de las componentes y la manera en que ellas se interrelacionan es motivo de debates teóricos sin una conclusión clara. Esto ocurre porque, en la realidad concreta y en relación con la manifestación de una conducta, las componentes operan conjuntamente dificultando la posibilidad de identificar su influencia de forma aislada. Como consecuencia, la perspectiva multidimensional presenta dificultades para ofrecer una síntesis que lleve a justificar por qué un alumno puede tener una actitud positiva o negativa (Di Martino \& Zan, 2001).

Aunque diferentes, las definiciones uni y multidimensionales no son contradictorias. En efecto, numerosos autores recurren a ambos niveles de abstracción para describir la estructura del constructo: un nivel más genérico en el que se capta la actitud en términos globales y otro nivel más específico en el que se admite una descomposición en componentes. Sin embargo, este es uno de los puntos de mayor desconexión entre las definiciones teóricas y las operacionales del constructo, dado que en los estudios cuantitativos rara vez se contrasta empíricamente una estructura jerárquica (Palacios, Arias, \& Arias, 2014).

\section{Dimensiones de la actitud hacia la matemática}

Los intentos por definir conceptualmente la actitud hacia la matemática y alcanzar mayor precisión en su marco teórico han sido desarrollados a través de dos abordajes 
metodológicos (Leder \& Forgasz, 2006; GómezChacón, 2002, 2010): a) el enfoque cualitativo, centrado en el estudio de manifestaciones afectivas en situaciones aúlicas y basados en el análisis del sujeto, desarrollando la actividad matemática en interacción con otros y b) el enfoque analítico-factorial, cuyo objetivo principal es la identificación de las dimensiones que forman parte de la estructura interna del constructo.

$\mathrm{Al}$ igual que con otros constructos tradicionales de la psicología como la personalidad o la inteligencia, el enfoque analítico-factorial se abrió paso para alcanzar una delimitación conceptual de la actitud hacia la matemática. Las pruebas desarrolladas en las últimas décadas tienen en común el uso del análisis factorial para identificar las dimensiones subyacentes a la variable y así obtener evidencias de validez teórica. Desde esta aproximación se tendió a definir este constructo, en ocasiones de manera implícita, a partir de los instrumentos construidos para su medición (Neale, 1969), generando importantes críticas sobre la vaguedad y ambigüedad de la teoría (Di Martino \& Zan, 2015; Hannula, 2002). No obstante, se ha tenido una prolífica producción de instrumentos con operacionalizaciones unidimensionales (Brito, 1998; Rodríguez-Jiménez \& Mora Mojica, 2016; Vendramini, Silva, \& Dias, 2009) y multidimensionales (Aiken, 1974; Auzmendi, 1992; Bazán \& Sotero, 1998; Fennema \& Sherman, 1976; Palacios et al., 2014; Tapia \& Marsh, 2004).

El aspecto más controvertido de la aproximación analítico-factorial es la falta de acuerdo sobre cuántas y cuáles son las dimensiones que componen la actitud hacia la matemática. La delimitación conceptual del constructo varía en función de la cantidad de componentes identificadas por los autores de los diferentes instrumentos. En una sistematización realizada por Muñoz y Mato (2008), se reportó que las dimensiones más frecuentemente reportadas en la bibliografía son: Agrado, Ansiedad, Utilidad, Confianza y Motivación.

\section{Agrado o afecto}

La dimensión afectiva-emocional se encuentra reflejada en gran parte de los modelos teóricos sobre las actitudes y ocupa un lugar preponderante en las teorías unidimensionales. Hace referencia al conjunto de sentimientos $\mathrm{y}$ emociones que experimenta el individuo al enfrentarse con la matemática. Las definiciones de esta dimensión aluden principalmente al agrado o disfrute que percibe el estudiante en el trabajo con la matemática y su estudio (Aiken, 1974; Adelson \& McCoach, 2011; Auzmendi, 1992; Bazán \& Sotero, 1998; Palacios et al., 2014; Tapia \& Marsh, 2004).

\section{Ansiedad}

Dentro del conjunto de indicadores que remiten a las emociones, aquellos que se refieren a la expresión de ansiedad ante la matemática demostraron ocupar un espacio importante en la conceptualización de este constructo. Numerosos estudios empíricos encontraron que estos indicadores se aislaron factorialmente, conformando una dimensión independiente (Auzmendi, 1992; Bazán \& Sotero, 1998). En estos casos, los test construidos recurrieron a manifestaciones fisiológicas, conductuales y cognitivas de la ansiedad con el fin de detectar niveles que obstruyan el aprendizaje de la matemática.

\section{Utilidad}

$\mathrm{Si}$ bien existen ligeros matices entre las distintas definiciones, los autores coinciden en caracterizar la Utilidad como las creencias sobre los beneficios o productividad que puede dar el conocimiento matemático para la vida académica y/o profesional del estudiante. Los apelativos más usados para su medición fueron Utilidad (Auzmendi, 1992; Fennema \& Sherman, 1976; Palacios et al., 2014), Aplicabilidad (Bazán \& Sotero, 2000) y Valor (Tapia \& Marsh, 2004). 


\section{Confianza}

Este aspecto se refiere al conjunto de creencias del estudiante sobre sus posibilidades y dificultades para responder a las habilidades requeridas en la actividad matemática. Esta dimensión recibió diversas denominaciones: Actitud hacia el éxito en matemática (Fennema \& Sherman, 1976), Autopercepción (Adelson \& McCoach, 2011), Habilidad (Bazán \& Sotero, 1998), Autoconcepto matemático (Palacios et al., 2014) y Confianza (Auzmendi, 1992; Tapia \& Marsh, 2004).

\section{Motivación}

La mayoría de los autores describen esta dimensión como el interés del estudiante por la matemática (Auzmendi, 1992; Fennema \& Sherman, 1976; Tapia \& Marsh, 2004). El problema que presenta la operacionalización de este constructo es que los indicadores utilizados se solapan con otras dimensiones. La principal dificultad se observa en que los ítems que describen la motivación intrínseca aluden a afectos positivos o negativos (componente Agrado) que pueden aparecer en la interacción con la matemática, mientras que aquellos que aluden a motivación extrínseca remiten al componente Utilidad.

\section{Método}

\section{Diseño}

El estudio realizado responde a las características de un estudio instrumental conforme a la clasificación de diseños de investigación en Psicología más recientes (Ato, López, \& Benavente, 2013; Montero \& León; 2007). Se trata de un desarrollo de corte psicométrico centrado en la fase de validez interna-estructural de la construcción de un instrumento de medida (Loevinger, 1957). En consecuencia, no es posible plantear las tradicionales preguntas de investigación o hipótesis de trabajo propias de una investigación empírica.

\section{Participantes}

Se contó con la colaboración de 2814 estudiantes de la carrera de Psicología de la Universidad de Buenos Aires. El 82 \% fueron mujeres y el 18 $\%$ fueron varones. La edad de los participantes osciló entre 18 y 62 años, con una media de 22.7 años $(D E=6.33)$, una mediana de 20, una amplitud semi-intercuartil de 2 años y un coeficiente de asimetría de Fisher de 3.06.

Procedimiento y análisis de datos

\section{Elaboración de la versión preliminar}

Se construyó un mapa del constructo a partir de las dimensiones más frecuentemente reportadas en la bibliografía (Afecto, Ansiedad, Utilidad, Confianza y Motivación). Los 75 ítems (15 por dimensión) se obtuvieron considerando dos fuentes principales: a) 48 indicadores procedentes de diversos instrumentos ya elaborados que miden el mismo constructo en población general o actitudes hacia objetos similares (Aiken, 1974; Auzmendi, 1992; Fennema \& Sherman, 1976; Tapia \& Marsh, 2004) y b) 27 indicadores que son originales y surgieron como resultante de un proceso inductivo a posteriori, que comenzó con entrevistas de grupo informales a estudiantes con características similares a los sujetos que formaron la población meta. El formato de respuesta tipo Likert de los ítems tiene seis categorías (desde Totalmente en desacuerdo a Totalmente de acuerdo).

\section{Depuración primaria}

Los ítems se sometieron al juicio de expertos en psicometría y profesores de Estadística de la carrera de Psicología. Solo fueron conservados los ítems para los que todos los jueces acordaron sobre la relevancia del indicador, la congruencia 
con la definición de la componente medida y la pertinencia de sus características formales. Esta información fue complementada con un estudio piloto, realizado con 32 estudiantes de Psicología representativos de la población meta. El objetivo de este estudio fue recabar información para eliminar los ítems que incluían palabras y expresiones que resultaban desconocidas $\mathrm{O}$ ambiguas para ellos.

\section{Administración de la versión depurada}

Los alumnos participaron de forma voluntaria y anónima con el fin de favorecer la sinceridad en las respuestas. Se realizó una charla motivadora previa para explicar el uso futuro de los resultados en investigación.

Análisis de items

Se dividió la muestra de manera aleatoria utilizando el $60 \%$ de la misma $(n=$ 1688), para efectuar una depuración del instrumento mediante un Análisis Factorial Exploratorio (AFE). Previo al AFE se eliminaron ítems que presentaron una baja frecuencia de elección (inferior al $2 \%$ ) en alguna de las categorías Likert o categorías extremas altamente atractivas (mayor al $40 \%$ ). Las respuestas del resto de los individuos $(n=$ 1126) fueron consideradas exclusivamente para el Análisis Factorial Confirmatorio (AFC) de los elementos que superaron el AFE. Ambos procedimientos factoriales se implementaron respetando el carácter ordinal de los datos, por lo que se basaron en las matrices de correlaciones policóricas. El AFE se aplicó operando el programa FACTOR (Lorenzo-Seva \& Ferrando, 2006). Se consideró el método de mínimos cuadrados no ponderados (ULS) para la extracción y la implementación óptima de análisis paralelo (Timmerman \& Lorenzo-Seva, 2011) como criterio para la retención de factores. El AFC se realizó mediante el programa Mplus (Muthén \& Muthén, 2010). Se estimaron los parámetros con el método de mínimos cuadrados ponderados robustos (WLSMV) y el ajuste se examinó usando el índice de ajuste comparativo (CFI), el índice de Tucker-Lewis (TLI) y el error medio cuadrático de aproximación (RMSEA).

\section{Estudios de confiabilidad}

Se analizó la consistencia interna de los factores mediante el alfa de Cronbach y el greatest lower bound (glb). También se estudió la estabilidad temporal de las puntuaciones con intervalo de 15 días. Para este estudio se consideró una submuestra aleatoria de los individuos $(\mathrm{N}=63)$ que participaron del AFC.

\section{Resultados}

\section{Depuración primaria}

El juicio experto permitió descartar un conjunto de 11 ítems (Tabla 1) que resultaban incongruentes con la definición operacional del constructo; eran indicadores que podían ser incluidos en otra dimensión para los que fueron propuestas o no representaban indicadores relevantes para estudiantes de un nivel universitario. Como resultado del estudio piloto solo fue descartado un ítem de Ansiedad que contenía la expresión sensaciones desagradables, considerada ambigua según la opinión de los evaluados. Ambos estudios aportan evidencias de validez de contenido y aparente.

\section{Tabla 1}

Cantidad de items eliminados en el proceso de depuración

\begin{tabular}{lrrrrrr}
\hline \multicolumn{1}{c}{ Estudio } & Afecto & Ansiedad & Utilidad & Confianza & Motivación & Total \\
\hline Juicio experto & 2 & 2 & 1 & 3 & 3 & 11 \\
$\begin{array}{l}\text { Piloto } \\
\begin{array}{l}\text { Distribuciones de } \\
\text { frecuencias }\end{array}\end{array}$ & - & 1 & - & - & - & 1 \\
$\begin{array}{l}\text { Análisis factorial } \\
\text { exploratorio }\end{array}$ & 7 & 4 & 1 & 1 & 4 & 12 \\
\hline Total eliminados & 11 & 13 & 7 & 9 & 11 & 51 \\
\hline
\end{tabular}

\section{Análisis Factorial Exploratorio}

De los 63 ítems administrados, 12 fueron eliminados antes de aplicar el AFE por presentar 
Facundo Juan Pablo Abal, Sofía Esmeralda Auné, Horacio Félix Attorresi.

distribuciones de frecuencias de la escala Likert marcadamente desbalanceadas ya sea por baja elección de una opción (inferior al $2 \%$ ) o categorías extremas atractivas (mayor al $40 \%$ ).

La implementación óptima del análisis paralelo de Horn realizado sobre la matriz de correlaciones de los 51 ítems sugirió la extracción de tres factores. Dado que los factores aislados mostraron una correlación considerable, se aplicó el método de rotación oblicua (Promax). La depuración de los ítems se llevó a cabo a partir de la información suministrada por la matriz de configuración. Sobre esta decisión, se efectuó la reducción de los ítems en etapas progresivas, aplicando tres criterios de eliminación: a) ítems con pesajes menores a 0.4 en todos los factores, b) ítems con pesajes superiores a 0.4 en dos o más factores y c) ítems propuestos para una dimensión teórica cuyo pesaje resultó superior a 0.4 en un factor diferente, salvo en el caso de que se pudiera justificar teóricamente el cambio de factor.

El resultado del proceso de depuración permitió alcanzar una solución factorial (KMO = 0.96; Test de Esfericidad de Bartlett: $\chi^{2}=$ $22923,1 ; g l=276 ; p<0.0001)$ rotada compuesta por 24 ítems distribuidos equilibradamente en tres factores que describieron el $63.7 \%$ de la varianza. La Tabla 2 resume las salidas finales obtenidas con la solución sin rotar y las matrices de configuración y estructura rotadas. El análisis de los residuos mostró un $\mathrm{RMSR}=0.0238$, el cual satisfizo el criterio de Kelley $(<0.0243)$.
Tabla 2

Resultados de los análisis factoriales exploratorio (AFE) y confirmatorio (AFC)

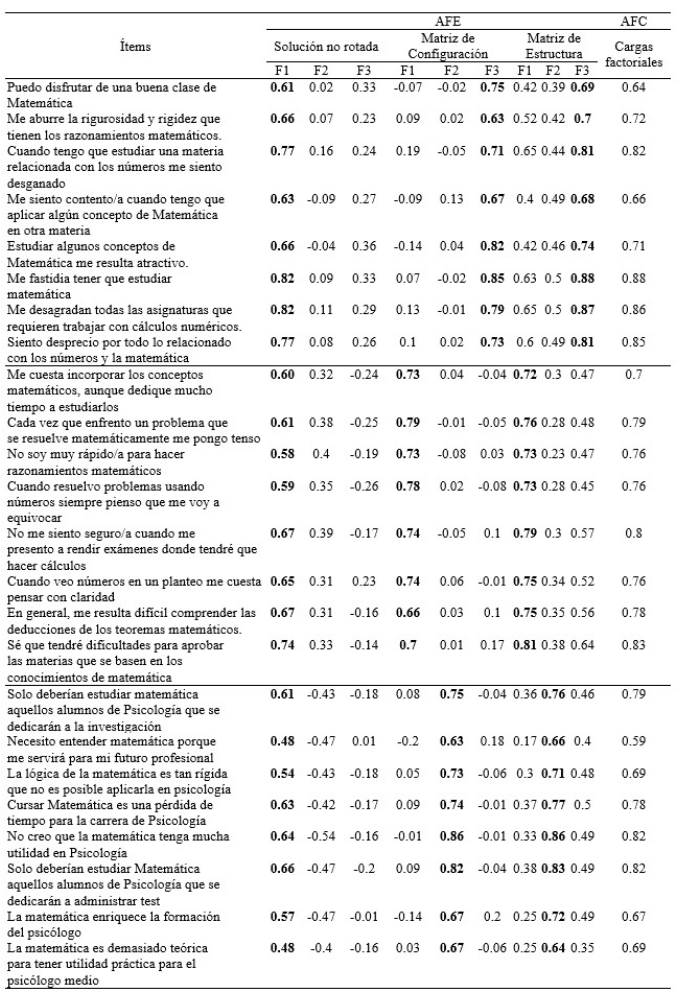

Los ítems que saturaron en el primer factor son indicativos, en su mayoría, de la dimensión Confianza y dos fueron planteados inicialmente como indicadores de Ansiedad. El segundo factor estuvo íntegramente compuesto por ítems redactados para la dimensión Utilidad. Por último, los elementos que componen el tercer factor provienen en cantidades iguales de las dimensiones Afectiva y Motivacional; es por esta razón que se denominó a este factor como componente Afectivo-motivacional.

\section{Análisis Factorial Confirmatorio}

Se sometió a prueba la estructura obtenida con el AFE (modelo de factores correlacionados), pero también se han propuesto otras estructuras alternativas que resultan interpretables a la luz de los hallazgos reportados en la bibliografía sobre el tema. Se examinó el ajuste de los datos a otros modelos frecuentemente reportados en la bibliografía: a) un modelo unidimensional 
que establece que los ítems se relacionan directamente con el constructo actitudinal global, b) un modelo en el que los tres factores aislados son independientes y c) un modelo jerárquico en el que los tres factores de primer orden definen un factor de segundo orden (modelo jerárquico).

La Tabla 3 exhibe los índices de ajuste obtenidos en los AFC para los diferentes modelos propuestos. Los resultados reflejan que ni el modelo unidimensional ni el modelo de tres factores independientes se ajustaron a los datos (TLI y CFI < 0.9; RMSEA > 0.08). Aun cuando el modelo jerárquico mostró un ajuste aceptable, se registró la estimación negativa de una varianza residual de un factor de primer orden. Por ende, su interpretación resulta inadmisible. Finalmente, el modelo que contempla los tres factores correlacionados es el único que mostró indicadores de ajuste adecuados, parámetros estimados significativos al $5 \%$ y los pesos estandarizados de cada uno de los ítems en sus respectivos factores fueron elevados (Tabla 2). El factor Afectivo-Motivacional presentó correlaciones moderadas tanto con Confianza $(r$ $=0.73 ; p<0.001)$ como con Utilidad $(r=$ 0.56; $p<0.001$ ). Estos dos últimos, en cambio, registraron una asociación menos intensa $(r=$ $0.38 ; p<0.001)$.

Tabla 3

Indicadores de ajuste del análisis factorial confirmatorio

\begin{tabular}{lccc}
\hline & CFI & TLI & RMSEA (IC 90\%) \\
\hline Tres dimensiones correlacionadas & 0.97 & 0.967 & $0.062(0.058-0.065)$ \\
Unifactorial & 0.742 & 0.717 & $0.181(0.177-0.184)$ \\
Tres dimensiones incorreladas & 0.648 & 0.615 & $0.211(0.208-0.214)$ \\
Jerárquico & 0.956 & 0.952 & $0.072(0.071-0.078)$ \\
\hline
\end{tabular}

\section{Estudios de confiabilidad}

Tanto los alfas de Cronbach como los glb resultaron igual o superiores a 0.9 dando cuenta de una alta consistencia interna de los ítems de cada factor. También resultaron satisfactorios los coeficientes de estabilidad temporal para las dimensiones Confianza $(\mathrm{r}=0.92, \mathrm{p}<0.001)$, Afectivo-Motivacional $(\mathrm{r}=0.85, \mathrm{p}<0.001)$ y Utilidad $(\mathrm{r}=0.83, \mathrm{p}<0.001)$.

| Universitas Psychologica | V. I 7 | No. 4 | 2018 |

\section{Comentarios}

La Psicología de la Educación y la enseñanza de la Matemática han tenido una producción abundante de instrumentos de evaluación pertenecientes a la dimensión afectiva del aprendizaje, y más específicamente, la actitud hacia la matemática (Carmona, 2004; Chamberlin, 2010; Leder \& Forgasz, 2006). A pesar de ser profusos los estudios que abordan las actitudes en el ámbito educativo, los resultados están lejos de ser concluyentes. Históricamente, la investigación cuantitativa sobre la actitud hacia la matemática se ha focalizado más en el desarrollo de los instrumentos de medida en lugar de priorizar la elaboración de una base teórica potente (Di Martino \& Zan, 2001, 2015). Es por esta razón que se busca que la aplicación de la metodología analítico-factorial usada en esta investigación sirva para optimizar el isomorfismo entre la realidad ofrecida por los datos empíricos y la concepción teórica del constructo.

La propuesta inicial sobre el constructo actitudinal se basó en un estudio de las cinco dimensiones más frecuentemente identificadas en la bibliografía. El proceso de validación del instrumento brindó un modelo de tres componentes para la población de estudiantes de Psicología: Afectivo-motivacional, Confianza y Utilidad. Una revisión de los ítems retenidos luego de la depuración permite concluir que no se ha afectado la validez de contenido examinada inicialmente. En este modelo, la Ansiedad no ocupa un puesto importante como en otras operacionalizaciones, sino que aparece subsumida en la dimensión Confianza. Posiblemente, la Ansiedad cobra mayor relevancia para poblaciones con nivel educativo secundario o en la transición al nivel universitario (Pérez-Tyteca, 2012).

Los estudios factoriales realizados permitieron especificar las características particulares que presenta la actitud hacia la matemática en la población analizada al tiempo que aportaron evidencias de validez de constructo. Otros investigadores también alcanzaron una estructura con tres componentes similares a los hallados en este trabajo. Pero resulta 
Facundo Juan Pablo Abal, Sofía Esmeralda Auné, Horacio Félix Attorresi.

difícil establecer puntos de comparación porque describen las actitudes de alumnos de nivel primario (Adelson \& McCoach, 2011) y estudiantes de ingeniería (Álvarez \& Soler, 2010). El modelo es asimilable incluso a las dimensiones Disposición Emocional (Afecto), Visión de la matemática (Utilidad) y Competencia percibida (Confianza) halladas por Di Martino y Zan (2010), utilizando la narrativa de los estudiantes como fuente de su estudio cualitativo.

La dimensión Confianza describe la percepción que tiene el estudiante de Psicología sobre su capacidad para enfrentarse con eficacia a situaciones que demandan el manejo de la matemática. Aquí se incluyen dos ítems pensados originalmente para la dimensión Ansiedad, pero que resultan congruentes con la definición de Confianza. Resulta razonable asumir que quienes experimentan malestar cuando deben aplicar conocimientos matemáticos perciban un menor nivel de seguridad en su desempeño. Numerosos autores han reportado este vínculo denominando como Autoconfianza (Tapia \& Marsh, 2004), Autoconcepto negativo (Alemany \& Lara, 2010) y Seguridad (Darías Morales, 2000) a esta dimensión. La dimensión Utilidad permite medir un conjunto de creencias con respecto a la relación entre la psicología y la matemática, así como también la aplicabilidad de las nociones matemáticas durante la formación académica y en el futuro desempeño profesional. Los ítems de esta dimensión han mostrado un elevado grado de homogeneidad durante todo el proceso de depuración. Finalmente, la dimensión Afectivo-motivacional refleja la tendencia de los alumnos a interesarse por la matemática en la medida que experimentan sentimientos positivos (o a registrar sentimientos agradables cada vez que se acercan a esta disciplina). Este hallazgo resulta consistente con algunos estudios en los que se han aislado factorialmente las dimensiones Afecto y Motivación, pero se registraron correlaciones superiores a 0.9 entre ellas (Lim \& Chapman 2013; Majeed, Ngurah, \& Lynch, 2013).

Al comparar las características del constructo actitud hacia la matemática delimitado en el presente trabajo con el de otras investigaciones aparece una distinción importante en relación con el método de rotación utilizado en el análisis factorial exploratorio de los ítems. Mientras que numerosos trabajos plantean una rotación ortogonal, y por ende, estructuras con factores incorrelados (Alemany \& Lara, 2010; Flores \& Auzmendi, 2015; Tapia \& Marsh, 2004) aquí se trabajó con rotación oblicua siguiendo las recomendaciones de Lloret-Segura, FerreresTraver, Hernández-Baeza y Tomás-Marco (2014). Las estructuras oblicuas proporcionan un marco más ajustado a la realidad para representar las interrelaciones de los componentes, aun cuando el resultado suponga una interpretación más compleja.

Si se consideran simultáneamente las variables del constructo actitudinal delimitado en este estudio, se puede asumir que las magnitudes de las correlaciones halladas entre los factores responden a lo esperable. Es decir, un estudiante con una actitud favorable tenderá a mostrar un afecto más positivo, una confianza más elevada y una mayor percepción de utilidad de la matemática. Sin embargo, los resultados obtenidos indican que esta tendencia no puede ser descripta por una estructura unidimensional de segundo orden. Es decir, la medición del constructo actitudinal se realiza a través de sus componentes, pero no se ha corroborado una concepción unitaria de la actitud hacia la matemática. En consecuencia, resulta insuficiente emplear la dicotomía negativopositivo para describir la actitud global como resultado de la integración de las componentes.

Como sugieren Di Martino y Zan (2010), parece razonable aceptar que la presencia de niveles bajos en cualquiera de los tres aspectos podría obstaculizar el proceso de aprendizaje. Los autores sugieren que una actitud puede ser considerada negativa si al menos una de las dimensiones resulta negativa; por lo tanto, recomiendan delinear diferentes perfiles que orienten las intervenciones de manera más específica. Por ejemplo, un alumno podría no manifestar rechazo hacia la matemática, sentirse seguro para llevar adelante la actividad matemática, pero cree que no es un 
conocimiento que le resulte útil para su desarrollo profesional en la psicología. El peso de esta creencia en su predisposición para construir el conocimiento que demanda la materia puede ser tan importante como el de otro estudiante que valore positivamente la aplicación de nociones matemáticas a la psicología, pero no se perciba capacitado para afrontar con éxito las exigencias. Aun así, se reconoce que la confianza en sí mismo para hacer frente a la matemática tiene una mayor estabilidad y resistencia al cambio que las ideas respecto de la utilidad, al menos en las condiciones contextuales en las que se lleva adelante el aprendizaje de esta materia en carreras como Psicología (Abal, Lozzia, Aguerri, Galibert, \& Attorresi, 2010).

Cabe señalar que la estructura del constructo actitud hacia la matemática obtenida en este estudio está circunscripta a las características singulares que presentan los estudiantes de Psicología. Esta es una fortaleza del instrumento, en tanto que resulta preciso para describir las diferencias de los individuos de esta población, pero también es una limitación dada su escasa posibilidad de generalización. Ulteriores estudios tendrán como objetivo realizar las adaptaciones correspondientes para intentar replicar esta estructura en estudiantes de otras carreras humanístico-sociales. Otra de las limitaciones de este trabajo es que solo se ha centrado en evidencias de validez basadas en la estructura interna de la escala. Futuras investigaciones buscarán evidencias de validez basadas en fuentes externas que permitan establecer cómo se vinculan las dimensiones con otros constructos o variables observables.

La actitud hacia la matemática es solo uno de los descriptores básicos de la dimensión afectiva del aprendizaje de la matemática. De ahí la necesidad de construir nuevos instrumentos y desarrollar otros dispositivos para captar el problema en su totalidad. Dadas las condiciones temporales y ambientales que se imponen en un curso de nivel universitario, parece razonable la recomendación de Leder y Forgasz (2006) acerca de evaluar los aspectos más estables del afecto (actitudes y creencias), mediante aproximaciones cuantitativas. En contraste, es aconsejable un estudio cualitativo de las emociones, porque son altamente dependientes de la situación donde se experimenta (McLeod, 1992). En definitiva, la complejidad del constructo demanda una metodología superadora que permita integrar los datos obtenidos a partir de estudios cualitativos y cuantitativos.

\section{Referencias}

Adelson, J. L., \& McCoach, D. B. (2011). Development and psychometric properties of the Math and Me Survey: Measuring third through sixth graders' attitudes towards mathematics. Measurement and Evaluation in Counseling and Development, 44(4), 225-247. http://dx.doi.org/10.1177/ 0748175611418522

Abal, F., Lozzia, L., Aguerri, M., Galibert, M., \& Attorresi, F. (noviembre, 2010). La utilidad de la matemática en el campo de la psicología desde la perspectiva de los estudiantes. Trabajo presentado en el II Encuentro de Docentes e Investigadores de Estadística en Psicología, Buenos Aires, Argentina.

Aiken, L. R. (1974). Two scale of attitude toward mathematics. Journal for Research in Mathematics Education, 5(2), 67-71. http:// dx.doi.org/10.2307/748616

Aiken, R. L., \& Aiken, D. R. (1969). Recent research on attitudes concerning science. Science Education, 53, 295-305. http://dx.d oi.org/10.1002/sce.3730530405

Alemany, I., \& Lara, A. I. (2010). Las actitudes hacia las matemáticas en el alumnado de la ESO: un instrumento para su medición. Publicaciones, 40, 49-71. Recuperado de http://revistaseug.ugr.es/index.php/publ icaciones/article/view/2228/2350

Álvarez, Y., \& Ruiz, M. (2010). Actitudes hacia las matemáticas en estudiantes de ingeniería en universidades autónomas venezolanas. Revista de Pedagogía, 31 (89), 225-249. Recuperado de http://www.scielo. org.ve/pdf/p/v31n89/art02.pdf 
Facundo Juan Pablo Abal, Sofía Esmeralda Auné, Horacio Félix Attorresi.

Ato, M., López, J. J., \& Benavente, A. (2013). Un sistema de clasificación de los diseños de investigación en psicología. Anales de Psicología, 29(3), 1038-1059. http://dx.doi. org/10.6018/analesps.29.3.178511

Auzmendi, E. (1992). Las actitudes hacia la matemática-estadística en las enseñanzas medias y universitarias. Bilbao: Mensajero.

Bazán, J., \& Sotero, H. (1998). Una aplicación al estudio de actitudes hacia la matemática en la UNALM. Anales Científicos UNALM, 36, 60-72. Recuperado de https://www.ime .usp.br/ jbazan/download/1998_62.pdf

Blanco, A. (2004). Enseñar y aprender estadística en las titulaciones universitarias de ciencias sociales. En J. C. Torre Puente \& E. Gil Coria (Eds.), Hacia una enseñanza universitaria centrada en el aprendizaje (pp. 143-190). Madrid: Universidad Pontificia Comillas.

Brito, M. (1998). Adaptação e validação de uma escala de atitudes em relação à Matemática. Zetetiqué, 6(1), 109-161. http://dx.doi.org/1 $0.20396 /$ zet.v6i9.8646811

Carmona, J. (2004). Una revisión de las evidencias de fiabilidad y validez de los cuestionarios de Actitudes y Ansiedad hacia la Estadística. Statistics Education Research Journal, 3(1), 5-28. Recuperado de https://pdfs.semanticscholar.org/2c89/d c5c9fdb8d927ee3762e5fb8bb11b70eb0f1.p df

Carpintero, H., Garriga Trillo, A. J., Ponsoda, V., \& Yela, M. (1989). Comentarios a la enseñanza de la Estadística en Psicología. Estadística española, 122, 476-495. Recuperado de http://www.ine.es/ss/Satellite?blobcol= urldata\&blobheader $=$ application $\% 2 \mathrm{Fpdf}$ \&blobheadername $1=$ Content - Disposition \&blobheadervalue $1=$ attachment $\% 3 \mathrm{~B}+$ fil ename\%3D297\%2F848\%2F122_3.pdf\&bl obkey $=$ urldata\&blobtable $=$ MungoBlobs \&blobwhere $=297 \% 2 F 848 \% 2 F 122$ 3.pdf \&ssbinary $=$ true

Chamberlin, S. (2010). A review of instruments created to assess affect in mathematics. Journal of Mathematics Education, 3(1),
167-182. http://educationforatoz.org/image s/14_Scott_A._Chamberlin.pdf

Coolican, H. (1994). Métodos de investigación y estadística en psicología. México: El Manual Moderno.

Cortada, N., Rodríguez Feijóo, N., \& Kohan Cortada, A. (noviembre, 2008). Análisis de las sugerencias para modificar las actitudes negativas hacia la estadística. Trabajo presentado en el I Encuentro de Docentes e Investigadores de Estadística en Psicología, Buenos Aires, Argentina.

Darias Morales, E. J. (2000). Escala de actitudes hacia la estadística. Psicothema, 12(2), 175-178. Recuperado de http://www.psicot hema.es/pdf/542.pdf

Di Martino, P., \& Zan, R. (julio, 2001). Attitude toward mathematics: some theoretical issues. En M. van den Heuvel-Panhuizen (Ed.), Proceedings of the 25th conference of the international group for the psychology of mathematics education (Vol. 3, pp. 351-358). Utrecht, The Netherlands: PME.

Di Martino, P., \& Zan, R. (2010). 'Me and maths': Towards a definition of attitude grounded on students' narratives. Journal of Mathematics Teacher Education, 13, 27-48. http://dx.doi.org/10.1007/s10857-0 09-9134-z

Di Martino, P., \& Zan, R. (2011). Attitude towards mathematics: A bridge between beliefs and emotions. Mathematics Education, 43(4), 471-482. http://dx.doi.or $\mathrm{g} / 10.1007 / \mathrm{s} 11858-011-0309-6$

Di Martino, P., \& Zan, R. (2015). The construct of attitude in mathematics education. En B. Pepin \& B. Roesken-Winter (Eds.), From Beliefs to dynamic affect systems in mathematics education (pp. 51-72). Nueva York: Springer. http://dx.doi.org/10.1007/9 78-3-319-06808-4

Fennema, E., \& Sherman, J. A. (1976). FennemaSherman Mathematics Attitudes Scales: Instruments designed to measure attitudes toward the learning of mathematics by females and males. Journal for Research in Mathematics Education, 7(5), 324-326. http ://dx.doi.org/10.2307/748467 
Flores, W. O., \& Auzmendi, E. (2015). Análisis de la estructura factorial de una escala de actitud hacia las matemáticas. Aula de Encuentro, 17(1), 45-77. Recuperado de https://revistaselectronicas.ujaen.es/ind ex.php/ADE/article/view/2256

Gairín, J. (1990). Las actitudes en educación. Un estudio sobre la educación matemática. Barcelona: Boixareu Universitaria.

García González, M. S., \& Juárez López, J. A. (2011). Revisión del constructo actitud en educación matemática: 1959-1979. Revista Iberoamericana de Educación Matemática, 26, 117-125. Recuperado de http://www.fisem.org/www/union/revist as/2011/26/archivo_12_de_volumen_26.p df

Goldin, G. A. (1988). Affective representation and mathematical problem solving. En M. J. Behr, C. B. Lacampagne \& M. M. Wheler (Eds.), Proceedings of the Tenth Annual Meeting on the Psychology of Mathematics Education, North American Chapter of International Group (pp. 1-7). North Illinois University: DeKalb, IL.

Gómez-Chacón, I. M. (2000). Matemática emocional. Los afectos en el aprendizaje matemático. Madrid: Narcea.

Gómez-Chacón, I. M. (2002). Cuestiones afectivas en la enseñanza de las matemáticas. Una perspectiva para el profesor. En L. C. Contreras \& L. J. Blanco (Eds.), Aportaciones a la formación inicial de maestros en el área de matemáticas: una mirada a la práctica docente (pp. 23-58). Cáceres: Universidad de Extremadura.

Gómez-Chacón, I. M. (2009). Actitudes matemáticas: propuestas para la transición del bachillerato a la universidad. Educación Matemática, 21 (3), 5-32. Recuperado de htt $\mathrm{p}: / /$ www.scielo.org.mx/scielo.php?script $=\mathrm{s}$ ci_arttext\&pid $=$ S1665-582620090003000 02

Gómez-Chacón, I. M. (2010). Tendencias actuales en investigación en matemáticas y afecto. En M. M. Moreno, A. Estrada, J. Carrillo \& T. A. Sierra (Eds.), Investigación en educación matemática XIV (pp. 121-140). Lleida: SEIEM.

Grootenboer, P., \& Marshman, M. (2016). Mathematics, affect and learning: Middle school students' beliefs and attitudes about mathematics education. Singapur: Springer.

Haladyna, T., Shaughnessy, J., \& Shaughnessy, M. (1983). A causal analysis of attitude toward mathematics. Journal for Research in Mathematics Education, 14(1), 19-29. http:/ /dx.doi.org/10.2307/748794

Hannula, M. S. (2002). Attitude towards mathematics: Emotions, expectations and values. Educational Studies in Mathematics, 49(1), 25-46. http://dx.doi.org/10.1023/A: 1016048823497

Harkness, S., D'ambrosio, B., \& Morrone, A. (2007). Preservice elementary teachers' voices describe how their teacher motivated them to do mathematics. Educational Studies in Mathematics, 65(2), 235-254. http://dx.doi.org/10.1007/s10649 $-006-9045-1$

Hart, L. (1989). Describing the affective domain: Saying what we mean. En D. Mc Leod \& V. M. Adams (Eds.), Affect and mathematical problem solving (pp. 37-45). Nueva York: Springer.

Hernández, G. (2011). Estado del arte de creencias y actitudes hacia las matemáticas. Cuadernos de Educación y Desarrollo, 3(24). Recuperado de http://www.eumed.net/rev/ ced/24/ghs.htm

Hidalgo, S., Maroto, A., \& Palacios, A. (2004). ¿Por qué se rechazan las matemáticas? Análisis evolutivo y multivariante de actitudes relevantes hacia las matemáticas. Revista de Educación, 334, 75-95. Recuperado de https://dialnet.unirioja.es/se rvlet/articulo? codigo $=963460$

Leder, G., \& Forgasz, H. J. (2006). Affect and mathematics education. En A. Gutiérrez \& P. Boero (Eds), Handbook of research on the psychology of mathematics education: Past, present and future (pp. 403-427). Nueva York: Sense Publishers.

Lim, S. Y., \& Chapman, E. (2013). Development of a short form of the attitudes toward 
Facundo Juan Pablo Abal, Sofía Esmeralda Auné, Horacio Félix Attorresi.

mathematics inventory. Educational Studies in Mathematics, 82, 145-164. http://dx.doi.o $\mathrm{rg} / 10.1007 / \mathrm{s} 10649-012-9414-\mathrm{x}$

Lloret-Segura, S., Ferreres-Traver, A., Hernández-Baeza, A., \& Tomás-Marco, I. (2014). El análisis factorial exploratorio de los ítems: una guía práctica, revisada y actualizada. Anales de Psicología, 30, 1151-1169. http://dx.doi.org/10.6018/anal esps.30.3.199361

Loevinger, J. (1957). Objective tests as instruments of psychological theory. Psychological Reports, 3, 635-694. http://d x.doi.org/10.2466/pr0.1957.3.3.635

Lorenzo-Seva, U., \& Ferrando, P. J. (2006). FACTOR: A computer program to fit the exploratory factor analysis model. Behavioral Research Methods, Instruments and Computers, 38(1), 88-91. http://dx.doi. org/10.3758/BF03192753

Majeed, A. A., Ngurah, I. G., \& Lynch, D. P. (2013). A confirmatory factor analysis of Attitudes toward Mathematics Inventory (ATMI). The Mathematics Educator, 15(1), 121-135. Recuperado de http://math.nie.ed u.sg/ame/matheduc/tme/tmeV15_1/7.pdf

Martínez, O. J. (2008). Actitudes hacia la matemática. Sapiens. Revista Universitaria de Investigación, 9(1), 237-256. Recuperado de https://dialnet.unirioja.es/servlet/articul o? codigo $=2781941$

McLeod, D. (1992). Research on affect in mathematics education: A reconceptualization. En D. A. Grouws (Ed.), Handbook of research on mathematics teaching and learning (pp. 575-596). Nueva York: MacMillan.

Montero, I., \& León, O. G. (2007). Sistema de clasificación del método en los informes de investigación en psicología. International Journal of Clinical and Health Psychology, 7(3), 847-862. Recuperado de http://www. aepc.es/ijchp/articulos_pdf/ijchp-256.pdf

Muñoz, J. M., \& Mato, M. D. (2008). Análisis de las actitudes respecto a las matemáticas en alumnos de ESO. Revista de Investigación Educativa, 26(1), 209-226. Recuperado de http://www.redalyc.org/pdf/2833/28332 1884011.pdf

Muthén, L., \& Muthén, B. (2010). Mplus User'sGuide (6th. ed.) Los Angeles, CA: Muthén \& Muthén.

Narro, A. E. (1997). Investigación sobre la concepción de la matemática en ciencias sociales en la UAM-Xochimilco. Política y Cultura, 9, 249-280. Recuperado de http:// www.redalyc.org/articulo.oa?id $=26700914$

Neale, D. (1969). The role of attitudes in learning mathematics. The Arithmetic Teacher, 16(8), 631-641. Recuperado de http://www.jstor.o $\mathrm{rg} / \mathrm{stable} / 41187564$

Palacios, A., Arias, V., \& Arias, B. (2014). Attitudes towards mathematics: construction and validation of a measurement instrument. Revista de Psicodidáctica, 19(1), 67-91. http://dx.doi.o $\mathrm{rg} / 10.1387 /$ RevPsicodidact.8961

Pérez-Tyteca, P. (2012). La ansiedad matemática como centro de un modelo causal predictivo en la elección de carreras (Tesis doctoral inédita). Universidad de Granada, España. Recuperada de http://hera.ugr.es/tesisugr/2 108144x.pdf

Rodríguez Feijóo, N. (2011). Actitudes de los estudiantes universitarios hacia la estadística. Interdisciplinaria, 28(2), 199-205. Recuperado de http://www.scielo. org.ar/pdf/interd/v28n2/v28n2a02.pdf

Rodríguez Jiménez, O., \& Mora Mojica, S. (2016). Análisis psicométrico con el modelo de respuesta graduada de Samejima. Actualidades en Psicología, 30(120), 7-30. http://dx.doi.org/10.15517/a p.v30i120.18722

Ruiz de Miguel, C. (2015). Actitudes hacia la estadística de los alumnos del grado en pedagogía, educación social y maestro de educación primaria en la UCM. Educación XXI, 18(2), 351-374. http://dx.doi.org/10.5 944/educXX1.12158

Sánchez Mendías, J. (2013). Actitudes hacia la matemática de los futuros maestros de educación primaria (Tesis doctoral inédita). Universidad de Granada, España. 
Recuperada de http://digibug.ugr.es/bitstre am/10481/29827/1/2194717x.pdf

Tapia, M., \& Marsh, G. E. (2004). An instrument to measure mathematics attitudes. Academic Exchange Quarterly, 8(2), 16-21. Recuperado de http://www.rap idintellect.com/AEQweb/cho25344l.htm

Timmerman, M. E., \& Lorenzo-Seva, U. (2011). Dimensionality assessment of ordered polytomous items with parallel analysis. Psychological Methods, 16, 209-220. http://d x.doi.org/10.1037/a0023353

Vendramini, C. M. M., Silva, M. C. R., \& Dias, A. S. (2009). Avaliação de atitudes de estudantes de psicologia via modelo de crédito parcial da TRI. Psico-Usf, 14(3), 287-298. http://dx.doi.org/10.1590/S141382712009000300005

\section{Notas}

* Artículo de investigación. Esta investigación fue financiada con subsidios de la Universidad de Buenos Aires (UBACyT 2014-17, Código 20020130100320BA) y de la Agencia Nacional de Promoción Científica y Tecnológica (ANPCyT PICT 2011 № 0826). 